\title{
In vivo aroma release measurements and sensory perception of flavoured milk proteins gels
}

Gierczynski, I.*, Labouré, H. and Guichard, E.

Unité Mixte de Recherche Flaveur, Vision, et Comportement du Consommateur ENESAD-INRA, 17 rue Sully, BP 86510, F-21065 Dijon, France

*Corresponding author: Isabelle.Gierczynski@dijon.inra.fr

\begin{abstract}
Three mixed milk gels with different structures were formulated. The addition of increasing concentration of chymosin resulted in an increase in gel hardness evaluated by penetrometry measurements. The in vivo release of aroma compounds from these gels was studied by Atmospheric Pressure Chemical Ionisation - Mass Spectrometry (APCI-MS) in Nosespace configuration and with a panel of fourteen subjects. Aroma release was found to be strongly affected by structure variation. An increase in gel hardness resulted in an increase in the amount and the rate of aroma release. In parallel, sensory analysis was performed using the profile method. Data treatment revealed that the different structures were perceived at the sensory level and could be described with numerous texture attributes. Intensity of salt perception was found to decrease from the softer to the harder gel. Overall aroma perception presented a tendency to decrease with gel hardness. Aroma perception could not be correlated with aroma release parameters, and cross-modal interactions were not excluded to explain results.
\end{abstract}

Keywords: Atmospheric Pressure Chemical Ionisation-Mass Spectrometry; APCI-MS; aroma release; milk gels; sensory perception; texture; taste.

\section{INTRODUCTION}

Texture, taste and aroma play a major role in the multidimensional sensory experience. They result from the stimulation of specific receptors and from human brain integration of all sensory inputs. Aroma perception in particular results from the stimulation of olfactory receptors by aroma compounds released during the oral step of food consumption. The way in which flavour compounds are released from the food during eating is governed by numerous parameters: composition of the product (Guichard, 2002), structure of the product and its behaviour during mastication (Baines et al., 1987), partition of compounds responsible for the stimulation between food, saliva and gas phase (Taylor, 1998). Therefore, the understanding of human perception from the release characterisation remains a complex task.

In food industry, the modification of texture is commonly used for the formulation of new products. Texture can be changed by different ways, and the most common one consists in modifying the physico-chemical composition, as performed by the addition of thickeners. Otherwise the impact of these modifications is not well controlled. Indeed numerous studies have been dedicated to this problematic, and most of them showed that aroma release was not influenced by an increase in firmness although aroma intensity was significantly decreased by an increase in the firmness of the product (Weel et al., 2002). However other authors claimed that intensity of aroma perception could be related to the kinetic of release (Baek et al., 1999). Thus it is not clear yet to what extent aroma perception can be related to aroma release phenomena or can be modulated by other sensory dimensions perceived. In this context, our 
objective was to combine instrumental and sensory approaches to get further in the understanding of the mechanisms. To achieve this purpose, we designed an experimental plan to study the impact of structure variation on aroma release and sensory perceptions.

\section{MATERIAL AND METHODS}

Milk gels: preparation and properties.

Three model cheeses prepared from a combined acidification and renneting of a milk proteins dispersion were used. They were obtained after a three-steps preparation: i) reconstitution of a milk protein dispersion, ii) inoculation and aromatisation of the dispersion and iii) 24-h gelation period. This process allowed us to exactly control the composition of the products. The physico-chemical composition of the models, with the same concentration of other ingredients such as proteins and $\mathrm{NaCl}$, changed only in the amount of chymosin added (Table 1). Samples were aromatized with the same quantity of an aroma mixture formulated with ten aroma compounds: 3-methylbutanal, 1-octen-3-ol, heptan-2-one, nonan-2-one, hexanoic acid, ethyl butyrate, ethyl hexanoate, diacetyl, phenylacetaldehyde and octanal. Gels, stored at $4{ }^{\circ} \mathrm{C}$, were used until the fifth day after their preparation.

Rheological measurements were performed to study the repeatability of the manufacture process and to characterize mechanical properties of the gels. Gels behaviour submitted to large deformation were studied by penetrometry tests. Experiments were conducted at $5{ }^{\circ} \mathrm{C}$ using a TA-XT2 Texture Analyser (Stable Micro Systems) fitted with a $10-\mathrm{mm}$ diameter cylinder probe at a constant penetration speed of $1 \mathrm{~mm} \cdot \mathrm{s}^{-1}$ and until deformation reached $30 \%$ of the initial height of the samples. Ten replicates of the measurements were performed. From the force-deplacement curve, we extracted the slope of the curve between 0 and $2 \mathrm{~mm}$ which provides the modulus of elasticity value (E), and the force at the maximum peak (Fmax) which represents the hardness of the gel. Fmax values are reported in Table 1 and show that the three gels have significant different properties. In particular the addition of chymosin results in an increase in gel hardness.

During the gelation period, the continuous acidification induced by starter cultures was followed by $\mathrm{pH}$ recording (PHM210 pH-meter and pHC3031-9 combined electrode, Radiometer Analytical). As shown in Table 1, this control was useful to check that products had the same $\mathrm{pH}$.

\begin{tabular}{|c|c|c|c|}
\hline \multicolumn{4}{|c|}{ Composition per $1 \mathrm{~kg}$} \\
\hline & M0 & M3 & M10 \\
\hline Micellar casein (g) & & 75 & \\
\hline whey protein $(\mathrm{g})$ & & 25 & \\
\hline Lactose $(\mathrm{g})$ & & 40 & \\
\hline $\mathrm{NaCl}(\mathrm{g})$ & & 10 & \\
\hline Yeast extract (g) & & 2 & \\
\hline Mineral solution $(\mathrm{mL})$ & & 1 & \\
\hline Starter culture $(\mathrm{mL})$ & & 0.2 & \\
\hline Chymosin $(\mu \mathrm{g})$ & 0 & 3 & 10 \\
\hline Blend of aroma $(\mathrm{mL})$ & & 0.9 & \\
\hline \multicolumn{4}{|c|}{ Gel properties } \\
\hline & M0 & M3 & M10 \\
\hline $\operatorname{Fmax}(\mathrm{N})$ & $0.5^{\mathrm{c}}$ & $1.5^{\mathrm{b}}$ & $2^{\mathrm{a}}$ \\
\hline pH & & 4.6 & \\
\hline
\end{tabular}

Table 1: composition, Fmax and pH values of the three model cheeses. For Fmax values, letters (a-c) indicate that means were different at $\mathbf{p}<\mathbf{0 . 0 5}$ using the Student-Newman-Keuls test. 


\section{In vivo aroma release measurements.}

A panel of fourteen subjects participated to nosespace measurements which consist in monitoring part of subject's breath composition. In this way we are able to describe aroma compounds release in real time. Breath-by-breath aroma release measurements were carried out using an ion trap mass spectrometer Esquire-LC (Bruker Daltonique, Wissembourg, France) equipped with a home-built interface designed to optimise gaseous sampling through a Venturi effect (Sémon et al., 2003). To optimise the signal-to-noise ratio, inlet flow rate was precisely adjusted to $30 \mathrm{~mL} / \mathrm{min}$.

Experiments were performed with the following fixed eating protocol. First subjects were asked to place the glass tube allowing sampling in one nostril and to breath regularly during $30 \mathrm{~s}$. This period was used to check the absence of residues of the previous sample in the subjects' breath. They were then instructed to take in their mouth the sample presented in a spoon, and to keep their mouth closed for $12 \mathrm{~s}$. Then the subjects were asked to chew the product during $20 \mathrm{~s}$ while keeping their mouth closed and without swallowing (chewing phase). Finally they were asked to swallow the bolus and to continue the test during $36 \mathrm{~s}$ (swallowing phase). During this phase, subjects were allowed to swallow as needed and they were also asked to disclose all their swallowing events (principal and secondary swallows) by taping with a stick. A total of eight replicates were obtained for each product and each subject.

Each individual raw release curve was first smoothed with a spline function, and different parameters were then extracted from the smoothed curves: AUCt, the area under the curve for the whole release which can be interpreted as the quantity of aroma released; AUC10, the area under the curve at $10 \mathrm{~s}$ of the release profile which can be considered as the slope of the curve and therefore can be related to the kinetic of release.

\section{Sensory analysis.}

Thirteen subjects of the APCI-MS panel participated to a sensory study performed to describe the perceptions of texture, taste and aroma generated from the consumption of our products. A complete profile was then established after an intense training. To follow the evolution of sensory perceptions throughout the breakdown of the product, the profile was performed at three times of the consumption: i) first compression between tongue and palate (time 1), ii) chewing period (time 2) and iii) analysis of the residual phase after the main swallowing of the bolus (time 3). Perceptions were also described during a second profile where subjects had nose clip. This part was used to investigate the effect of structure variation on taste perception by suppressing aroma perception.

\section{RESULTS}

\section{Impact of structure variation on aroma release.}

By APCI-MS technique, volatile compounds are ionized and detected at $m / z$ values corresponding in general to their protonated molecular ions $\left(\mathrm{MH}^{+}\right)$. But because of sensitivity problems only seven of the ten compounds present in aroma mixture could be analyzed: 3-methylbutanal $(\mathrm{m} / \mathrm{z} 69)$, 1-octen-3-ol $(\mathrm{m} / \mathrm{z} 111)$, heptan-2-one $(\mathrm{m} / \mathrm{z} 115)$, hexanoic acid and ethyl butyrate (both $\mathrm{m} / \mathrm{z} 117)$, nonan-2-one $(\mathrm{m} / \mathrm{z} 143)$ and ethyl hexanoate $(\mathrm{m} / \mathrm{z} 145)$. Diacetyl $(\mathrm{m} / \mathrm{z}$ 87) and phenylacetaldehyde $(\mathrm{m} / \mathrm{z} 121)$ were detected at low signal to noise ratios and thus were not studied during data analysis. Octanal $(\mathrm{m} / \mathrm{z} 111)$ was not observed, assuming that its concentration in the gel was too low. As aroma perception was described only with an overall intensity, results presented here concern the total signal resulting from the sum of the different ions contained between $\mathrm{m} / \mathrm{z} 110$ and $\mathrm{m} / \mathrm{z}$ 146. This interpretation was considered as valid because the same observations were made for each individual ion. 
For each extracted parameter, an analysis of variance was performed with the general linear model $(\mathrm{GLM})$ of SAS $($ model $=$ product + subject + session + product*subject + product*session + subject*session, and product effect tested against product*subject interaction). Significance of treatments was calculated with the LSMeans function of the GLM procedure. In each case, means were considered statistically different when $\mathrm{p}<0.05$. Statistical analysis revealed significant differences between products and results are summarized in Graphic 1.

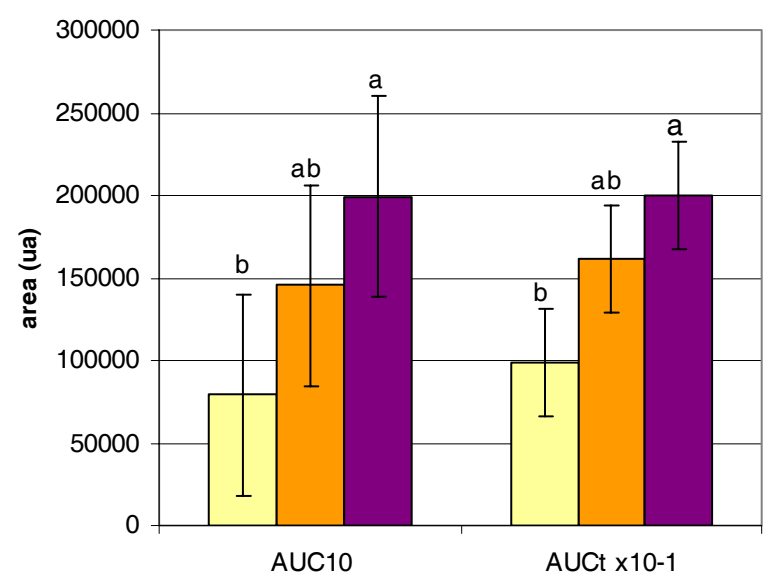

Graphic 1: In vivo aroma release parameters for M0 ( ), M3 ( $\square$ ) and M10 ( $\square)$. Mean values and 95\% confidence interval. Letters $(a, b)$ indicates that means are significantly different at $p<0.05$.

As shown in graphic 1, the amount of aroma release as well as the initial release rate is higher for M10 matrix and significantly increases with the firmness of the gel. As the composition of the three products (protein and aroma mixture content) is the same, we may suppose that data illustrate a structure impact and not physico-chemical interaction. A possible explanation for these differences may be a more extensive chewing for the most firm gel in favour of a more important quantity of aroma released. Data are in agreement with Boland et al. (Boland et al., 2006) who found an increase in the quantity of aroma released with the firmness of the gel. Moreover the increase in chymosin concentration has been showed to have a strong impact on gel structure. In particular the addition of chymosin leads to the formation of a stronger but more porous network (Lucey et al., 2001; Aichinger et al., 2003). The porosity of the gel could explain an easier release of aroma compounds resulting in higher release rates.

\section{Impact of structure variation on sensory perceptions.}

For each attribute, an analysis of variance was performed with the general linear model $(\mathrm{GLM})$ of SAS $($ model = product + subject + session + product*subject + product*session + subject*session, and product effect tested against product*subject interaction). Significance of treatments was calculated with the LSMeans function of the GLM procedure. Results are summarised in Graphic 2 and Graphic 3. 


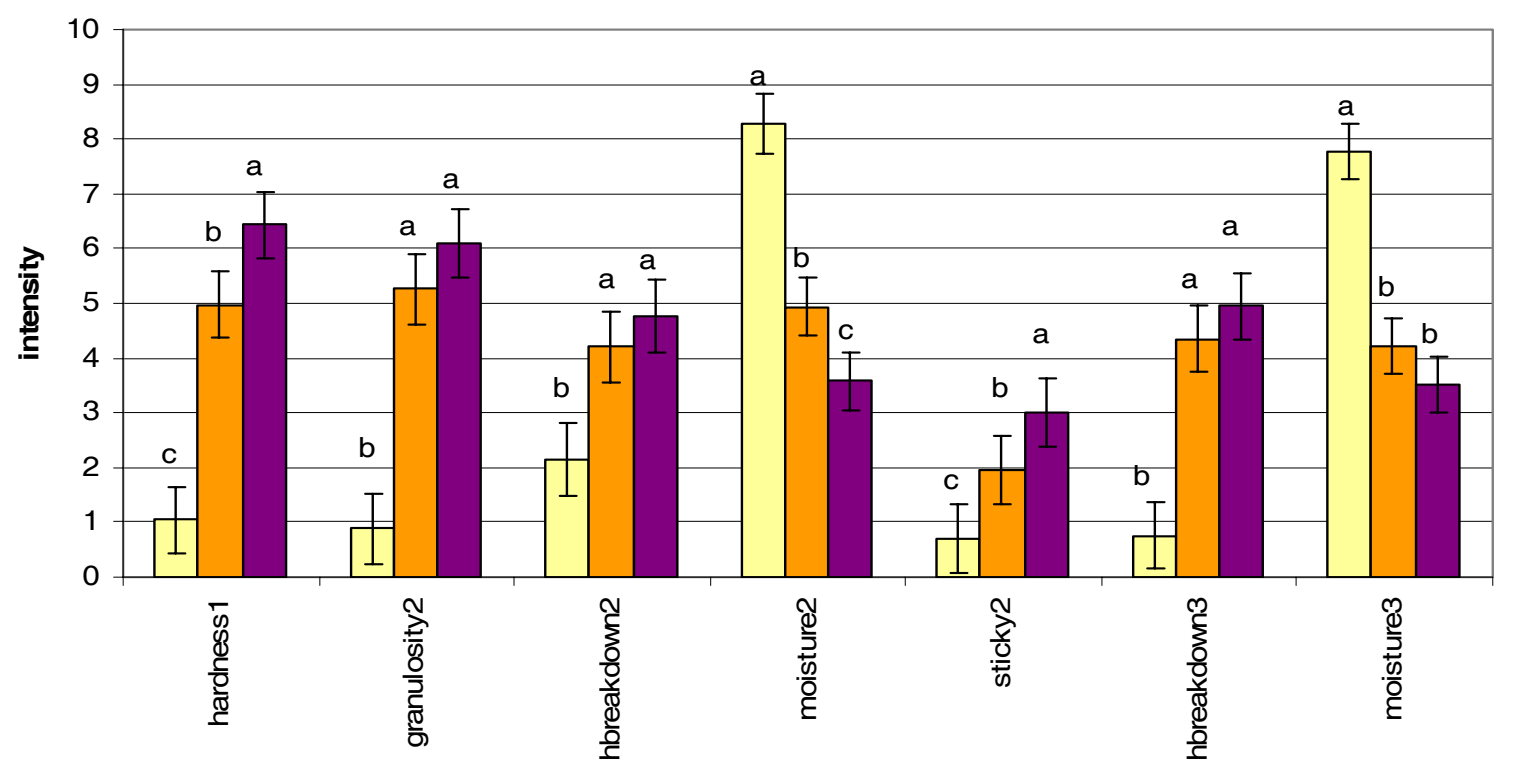

Graphic 2: Texture perception. Illustration of differences between products for M0 ( ), M3 ( $\square$ ) and M10 (口) : mean values and $95 \%$ confidence interval. Letters $(a-c)$ indicate that means are significantly different at $\mathbf{p}<\mathbf{0 . 0 5}$. The number $i$ after the descriptor indicates the evaluation time. hbreakdown is the abbreviation for the descriptor "heterogeneity of the breakdown".

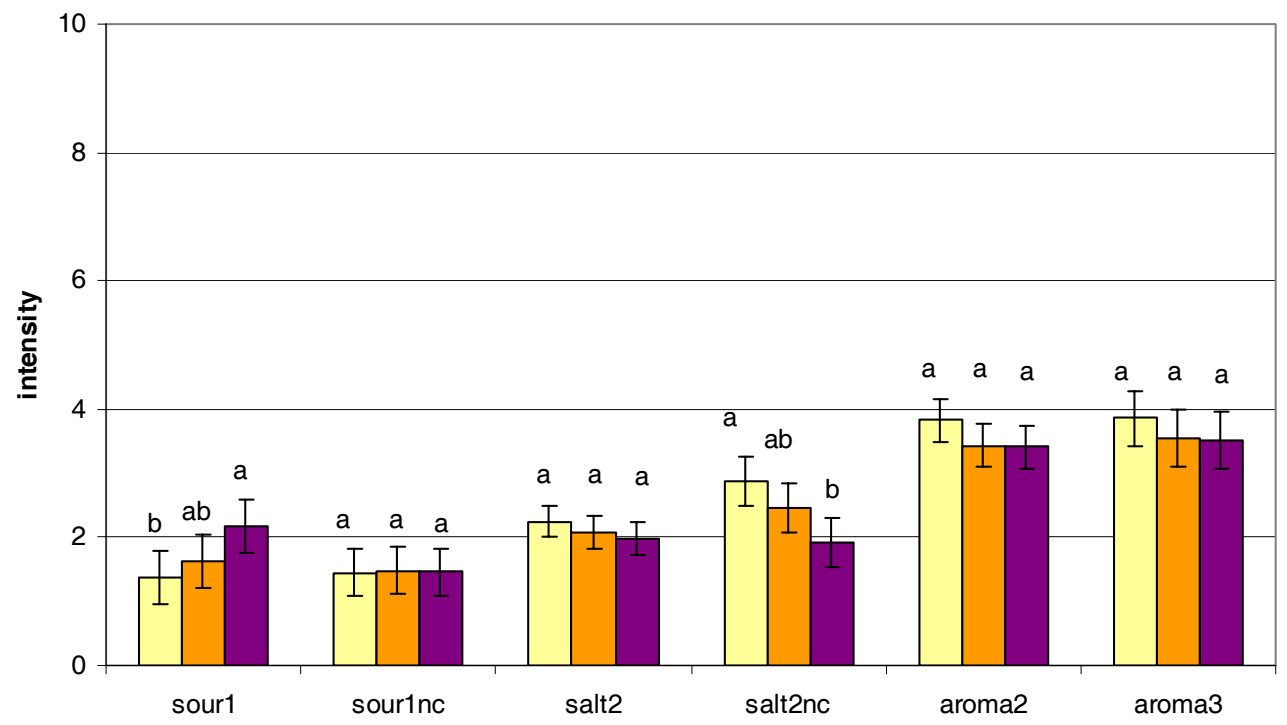

Graphic 3: Flavour perception. Illustration of differences between products for M0 ( ), M3 ( $\square$ ) and M10 (口) : mean values and $95 \%$ confidence interval. Letters $(a-c)$ indicate that means are significantly different at $p<0.05$. The number $i$ after the descriptor indicates the evaluation time. nc means that the descriptor was evaluated with nose clip.

The first analysis of sensory results shows that the most important differences between products were found for texture attributes and slighter differences were observed for flavour attributes. Indeed, for texture dimension, Graphic 2 shows that M3 and M10 gels have a heterogeneous breakdown and are perceived as harder, more granulous, stickier, and less humid. In other words, profile show that structural modifications induced by the addition of increasing concentrations of chymosin are perceived at the sensory level. As shown on Graphic 4, hardness perception presents the same tendency as rheological hardness determined through penetrometry measurements. Actually and logically, the highest Fmax value is, the hardest the gel is perceived. Differences on the heterogeneity of the breakdown 
can be interpreted as a consequence of gel structure. Indeed, gels made with both rennet gelation and acidification are formed with strong and resistant strands (Aichinger et al., 2003). We can therefore suppose that they are likely to break rather than to spread and that the subject describes this specific breakdown as heterogeneous. On the contrary, lactic gels are softer gels and will tend to flow during the chewing process, a breakdown which can be described as homogeneous. Finally we can observe that products are still perceived as different after they have been swallowed.

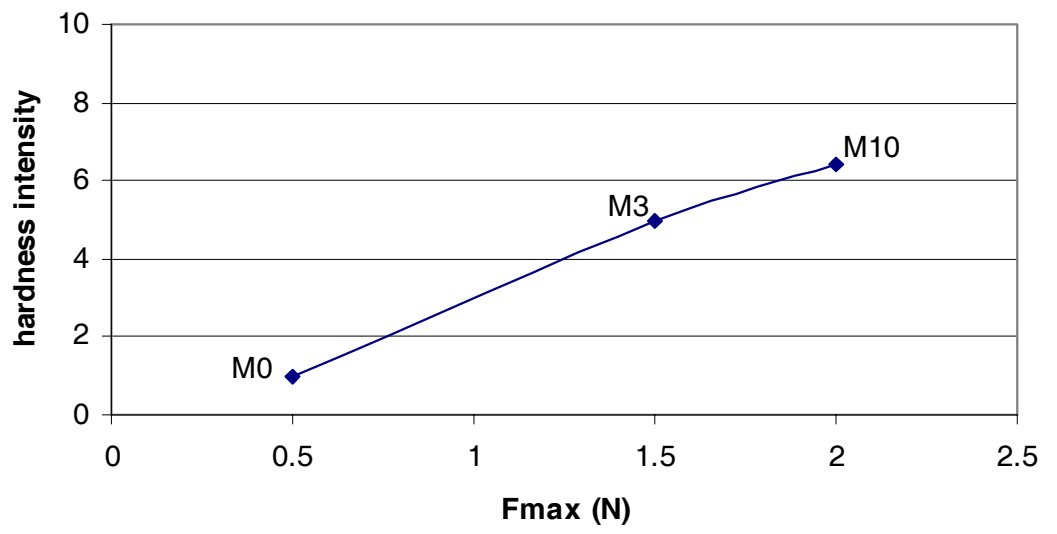

Graphic 4: Relationship between intensity of hardness perception and Fmax value determined through penetrometry measurements.

For taste dimension, Graphic 3 first reveals that taste attributes were not evaluated in a same way when aroma perception was present or not. Indeed sour intensity is significantly different between products only when subjects evaluate products without nose clip. Therefore we can suppose that sour intensity differences are due to aroma perception. M10 matrix, the hardest one, is perceived as sourer. This is coherent with the more intense and faster aroma release found by nosespace experiments. On the contrary, when aroma perception is suppressed, a significant effect of structure on salt perception appears, as if salt perception was partly masked by aroma perception. M0 matrix, the softer one, is perceived as more salty although the three matrices content the same level of $\mathrm{NaCl}$.

Finally, Graphic 3 shows that structure modification did not induce any variation of aroma perception intensity. However, further experiments conducted with a less intense and less complex aromatisation of the products highlighted a decrease in aroma intensity perceived with gel hardness although the same level of aroma mixture was added in the three products. Data are in contradiction with aroma release study which showed that the increase in gel hardness led to an increase in the amount and rate of aroma released. They are yet in agreement with previous studies who reported a decrease in intensity perceived with an increase in gel viscosity or gel hardness (Christensen, 1980; Cook et al., 2002; Hollowood et al., 2002; Lethuaut et al., 2004; Paçi Kora, 2004). Then, our results lead us to hypothesize that aroma perception is influenced through cross-modal interactions by the perception of texture and/or the perception of taste. In this particular case salt-aroma interactions may have occurred and the increase in salt perception could potentially account for the enhancement of aroma perception. But this hypothesis supposes that our salt/aroma association is congruent (Frank et al., 1988). Literature was used to tentatively explain the influence of structure variation on salt perception. Some authors supposed that the decrease in taste intensity with gel viscosity was due to a decrease in mass transfer of non volatile compounds from the product to the tongue (Baines et al., 1987), as a result of network formation as well as a less efficient mixing of the sample with saliva. In our case, we may suppose that the softer gel (M0) is quickly reduced in a viscous solution completely mixed with saliva and which form a 
coating film on the tongue. This behaviour would quickly stimulate all taste buds and this higher release rate could result in a higher intensity perceived. The other explanation is the possibility of texture influence on other sensory perception, as texture represents a sensory input on its own (Rolls et al., 2003). Mechanisms responsible for this influence have not been elucidated yet. We may for instance suppose that subjects are more concentrated on the texture perception of a firmer gel, as in particular it requires a more intense effort of mastication. This could therefore lead to disinterestedness to other perceptions which are under-evaluated.

\section{CONCLUSION}

An original way to formulate products with the same chemical composition but significant structures was used in order to investigate the impact of structure on aroma release and sensory perceptions. The addition of chymosin resulted in a different organisation of the network of flavoured milk gels, and the more important chymosin concentration was, the more important gel strength was. As a result of gel structure and gel breakdown during mastication, aroma release was found to be significantly influenced by structure modification. The increase in gel strength resulted in an increase in the amount and the rate of aroma release. Texture dimension of the profile evidenced the expression of the different structures at the sensory level. Intensity of salt perception, and to a lesser intensity of aroma perception, decreased from the softer to the harder gel, thereby providing the evidence of structure impact on sensory perceptions. The impact of structure on taste perception was supposed to originate from physico-chemical interactions, but aroma perception could not at all be related to aroma release factors. Therefore these results support the existence of cross-modal interactions between sensory modalities. However different situations were proposed, as a salt/aroma interaction or the influence of texture perception on aroma and/or taste perception.

\section{LITERATURE CITED}

Aichinger, P., Michel, M., Servais, C., Dillmann, M., Rouvet, M., D'Amico, N., Zink, R., Klostermeyer, H. and Horne, D. S. (2003). "Fermentation of a skim milk concentrate with Streptococcus thermophilus and chymosin : structure, viscoelasticity and syneresis of gels". Colloids and Surfaces B: Biointerfaces, 31, 243.

Baek, I., Linforth, R. S. T., Blake, A. and Taylor, A. J. (1999). "Sensory perception is related to the rate of change of volatile concentration in-nose during eating of model gels". Chemical Senses, 24(2), 155.

Baines, Z. V. and Morris, E. R. (1987). "Flavour/taste perception in thickened systems : the effect of guar gum above and below c". Food Hydrocolloids, 1(3), 197.

Boland, A. B., Delahunty, C. M. and van Ruth, S. M. (2006). "Influence of the texture of gelatin gels and pectin gels on strawberry flavour release and perception". Food Chemistry, 96(3), 452.

Christensen, C. M. (1980). "Effects of solution viscosity on perceived saltiness and sweetness". Perception \& Psychophysics, 28(4), 347.

Cook, D. J., Hollowood, T. A., Linforth, R. S. T. and Taylor, A. J. (2002). "Perception of taste intensity in solutions of random-coil polysaccharides above and below c*". Food Quality and Preference, 13(7-8), 473.

Frank, R. A. and Byram, J. (1988). "Taste-smell interactions are tastant and odorant dependent". Chemical Senses, 13(3), 445.

Guichard, E. (2002). "Interactions between flavor compounds and food ingredients and their influence on flavor perception". Food Reviews International, 18(1), 49. 
Hollowood, T. A., Linforth, R. S. T. and Taylor, A. J. (2002). "The effect of viscosity on the perception of flavour". Chemical Senses, 27(7), 583.

Lethuaut, L., Weel, K. G. C., Boelrijk, A. E. M. and Brossard, C. D. (2004). "Flavor perception and aroma release from model dairy desserts". Journal of Agricultural and Food Chemistry, 52(11), 3478.

Lucey, J. A., Tamehana, M., Singh, H. and Munro, P. A. (2001). "Effect of heat treatment on the physical properties of milk gels made with both rennet and acid". International Dairy Journal, 11, 559.

Paçi Kora, E. (2004). Thèse de Doctorat. Unité Mixte de Recherche Génie et Microbiologie des Procédés Alimentaires (INRA-INA PG). Paris, Institut National Agonomique Paris-Grignon.

Rolls, E. T., Verhagen, J. V. and Kadohisa, M. (2003). "Representations of the texture of food in the primate orbitofrontal cortex: Neurons responding to viscosity, grittiness, and capsaicin". Journal of Neurophysiology, 90(6), 3711.

Sémon, E., Gierczynski, I., Langlois, D. and Le Quéré, J. L. (2003). Advances in mass spectrometry. Ashcroft, A. E., Brenton, G. and Monaghan, J. J. (eds). Amsterdam, The Netherlands, Elsevier. 16, CD ROM Supplement.

Taylor, A. J. (1998). "Physical chemistry of flavour". International Journal of Food Science and Technology, 33(1), 53.

Weel, K. G. C., Boelrijk, A. E. M., Alting, A. C., vanMil, P. J. J. M., Burger, J. J., Gruppen, H., Voragen, A. G. J. and Smit, G. (2002). "Flavor release and perception of flavored whey protein gels: Perception is determined by texture rather than by release". Journal of Agricultural and Food Chemistry, 50(18), 5149. 